\title{
Water-Stress in Relation to Maize (Zea mays L.) Grain Yield, Plant Height and Proline Content
}

\author{
M.A.Gomaa ${ }^{1}$, I.F.Rehab ${ }^{1}$, F. A. Salama ${ }^{2}$ and A.S.M. AL-Deeb ${ }^{2}$ \\ ${ }^{1}$ Plant Production Dep. Faculty of agriculture, Saba Basha, Alex University \\ ${ }^{2}$ Mize Res. Prog., Field Crop Res. ARC, Egypt
}

\begin{abstract}
Two experiments were carried-out in Research Farm of Nubaria Agricultural Resear- ch Station (46 Km. south west of Alexandria), Agricultural Research Center, Ministry of Agri- cultural. In 2014 and 2016 seasons to study the potential of some maize genotype to waterstress through yield, yield components, some agrono- mic and physiological characteristics. Four maize hybrids (T.W.C321, S.C 162, S.C 10, S.C 129), its parent ( Gm. 2, Sd. 7, Sd. 63, Gz. 628, Gz

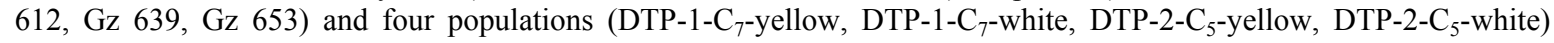
were grown in a split-plot design in 4 replicates under three irrigation treatments (control, moderate and severe ). Imposition of severe water treatment decreased the plant height and grain yield but increased proline content in leaves. Results showed signif-icant differences among genotypes and water deficiency treatments for grain yield and proline content.
\end{abstract}

Keywords: zea maize, water stress, grain yield, leaves proline content.

\section{INTRODUCTION}

Maize (Zea mays L.) is one of the most important food and feed crops in the world. In Egypt, it is used primarily as a feed crop and as industrial crop for oil and starch extraction. IN literature, maize has been reported as having high irrigation requirements (Rhoads and Bennett 1990; Stone et al. 2001). In arid and semi-arid regions, the daily evapotranspiration of maize often exceed 10 $\mathrm{mm}$ day $^{-1}$ for signif- icant time periods (Howell et al. 1995). Furthermore, maize is sensitive to water stress, especially at flowering and pollination stages. Nesmith and Ritchie (1992) reported that, the reductions in maize yield exceeded $90 \%$ due to water stress during flowering and pollination stages. Proline and quaternary ammonium com-pounds, e.g. Glycinebetaine, choline, proline betaine are keyosmolytes contributing osmotic adjustment (Huang et al., 2000 and Kavikishore et al., 2005).

Frederick et al. (1989) reported a decrease in maize yield due to drought stress associated with a number of barren plants, a lower number of kernels.ear ${ }^{-1}$ and a short grain filling period. Nigem (1998) reported positive and significant correlations between maize grain yield and each of the leaf area index, ear length and number of kernels row ${ }^{-1}$ under drought stress. Moursi (1997) reported that, under water stress condit-ions grain yield per faddan was positively correlated with ear weight and kernel weight/ear, therefore, he concluded that, grain yield of maize under stress conditions might be improved through selection for ear weight and kernel weight/ear. Ear weight was positively and significantly correlated with kernel weight/ear. Abd El-Gawadet al. (1980) investigates the effect of skipping one of six irrigations on yield of maize hybrid D.C. 355. They found that, grain yield, ear diameter, 100-kernel weight, ear number, and the percentage of double-eared plants were decreased. How-ever, the number of kernels/row was not affected. Skipping the third, fourth, or fifth irrigation reduced grain yield by $21,19.9$, and $17 \%$, respectively. Skipping the third or fourth irrigation decreased the number of ears/faddan. The greatest reduction in 100-kernel weight resulted from omitting the fifth or sixth irri-gation. Moustafa and Seif El-Yazal (1980) studied the effect of irrigation intervals ofnine, $12,15,18$, or 21 days at vegetative, flowering or maturity growth stages on hybrid D.C. 186. They found that grain yield was decreased with increased irrigation intervals at all growth stages. The greatest effect on grain yield was resulted from the effect on flowering stage. The best irrigation intervals of 12, nine, and 15 days were recommended, respectively, for vegetative, flowering, and maturing stages.

Porro and Cassel (1986) reported that delaying irrigation during a dry growing season reduced plant height.El-Ganayniet al. (2000) mentioned that, flowering stage was the most sensitive to water stress, where, the reduction was $67 \%$ in grain yield and $53 \%$ in number of ears per plant. They added that, grain-filling stage was also sensitive to water stress. The pre-flowering and flowering stages were equal in sensitivity to water stress effect on anthises to silking interval and number of rows/ear. When water stress elongated, it reduced number of rows/ear by $4 \%$ as compared to the control. Moreover, severe stress experienced from the beginning of flowering stage until maturity showed maximum reductions in grain yield (75\%), ears/plant (56\%), and rows/ear (5\%) as compared to the control. Also, prolonged irrigation interval of 22 days significantly reduced grain yield/plant and 
number of kernels/ear as compared to irrigation at 12-days. Reduction occurred also in plant height, ear height, and leaf area. Asch et al., (2001), found that, plant height was significantly reduced by 40 and $25 \%$, respectively, in the two most severe drought treatments. Long drying cycles resulted in significant yield reductions up to $70 \%$ of the fully watered controls. Kernel number per cob was reduced up to $60 \%$ under long drought conditions and not affected under short-term drought.

Soltani, et al (2013), showed that, water deficiency induced significant increase of leaves proline. Also, Water deficiency led to significant decrease in chlorophyll content. Efeoĝlu, et al, (2009), found that, the Fresh and dry biomass, fluorescence and Chlorophyll decreased with drought but the proline contents was increased. Tarighaleslami, et al (2012), found that Proline also increased significantly under drought stress conditions showing that osmotic adjustment mechanism had been activated. Lama, and Chakraborty, (2013), Showed that, with increasing in the intensity of drought there was an increase in both proline and ascorbate content. Heidari, and Moaveni, (2009), indicated that, drought stress affected different activity levels of the proline and abscisic acid (ABA).

The aim of this study was to investigate the effect of water stress treatments on yield, yield components and some agronomic characteristics using 4 hybrids with its parents and four populations (white and yellow) in the two summer growing seasons (2014-2016).

\section{MATERIAL AND METHODS}

The present study was carried-out at Nubaria Agricultural Research Station (46 Km. south west of Alexandria), Agricultural Re-search Center, Ministry of Agricultural, Egypt. The site of experiment was chosen to represent irrigation problem in newly reclaimed lands of Nubaria region with soil $\mathrm{PH} 8.4$,organic matter $0.60 \%, \mathrm{CaCO}_{3}$ $31.8 \%$, and electrical conductivity of $0.55 \mathrm{ds} \cdot \mathrm{m}^{-1}$. The experimental design was a split-plot with 4 replicates. Irrigation treatments (10 days, 15 days and 20 days) were assigned to main plots. Genotypes used in this study were four commercial hybrids, their parents, and four populations are shown in table (1), and were assigned to sub-plot. The sub-plot consisted of four rows of three meter long and $0.7 \mathrm{~m}$ apart. Two central rows were harvested for yield and yield components data. Sowing date was normal at the two successive seasons (2014 and 2016), respectively. Two seeds were hand sown per hill. Spaced at $25 \mathrm{~cm}$. Hills were thinned to one plant/hill after 21 days from planting. Nitrogen fertilization at rat of $120 \mathrm{~kg} / \mathrm{fad}$ (ammonium nitrate 33.5) was applied in two equal doses before the first and second irrigation. Harvest was done after 120 days from sowing.

\section{The study characters were}

1-Plant height $(\mathrm{cm})$ : measured from ground to the point of flag leaf insertion.

2- Grain yield plant ${ }^{-1}(\mathrm{~g})$ adjusted at $15.5 \%$ grain moisture.

Table 1: The lines, hybrids and drought tolerant populationswere used in this study.

\begin{tabular}{|c|c|c|c|c|}
\hline Genotypes & Abridged & Origin & Color & Pedigree \\
\hline \multicolumn{5}{|c|}{ Lines } \\
\hline Gemmeiza 2 & $\mathrm{Gm}-2$ & Mexican & white & Pop. 7421 CIMMYT (Pop21) \\
\hline Sides 7 & $\mathrm{Sd} 7$ & ARC & white & A.E.D $\times$ an exotic composite, A4 \\
\hline Sides 63 & Sd 63 & Mexican & white & Teplacinco \# 5 (Tep-5) \\
\hline Giza 612 & Gz 612 & ARC & white & B73 (P-90 Bsss-1) x Sd7 \\
\hline Giza 628 & Gz 628 & $\mathrm{ARC}$ & white & B73 (P-90 Bsss-1) x Sd-62 \\
\hline Giza 639 & Gz 639 & $\mathrm{ARC}$ & yellow & B73 (P-90 Bsss-1) xSd 62( $\left(\mathrm{s}_{5}\right)$ \\
\hline Giza 653 & Gz 653 & ARC & yellow & EXP 9281 \\
\hline \multicolumn{5}{|l|}{ Hybrid } \\
\hline Single cross 10 & S.C. 10 & ARC & white & $\mathrm{Sd}-7 \mathrm{X} \mathrm{Sd}-63$ \\
\hline Single cross 129 & S.C. 129 & $\mathrm{ARC}$ & white & $\mathrm{Gz}-612 \times \mathrm{Gz}-628$ \\
\hline Single cross 162 & S.C. 162 & ARC & yellow & $\mathrm{Gz}-653$ X Gz-639 \\
\hline Three way cross 321 & T.W.C. 321 & ARC & white & {$[$ S.C.21(Gm-2 X Sd 63)] X Sd - 7} \\
\hline \multicolumn{5}{|c|}{$\begin{array}{r}\text { Population } \\
\end{array}$} \\
\hline Drought tolerant population one & DTP-1-C 7 (W1) & ARC & white & TL 95b-6677/9 \\
\hline Drought tolerant population one & DTP-1- $\mathrm{C}_{7}(\mathrm{Y} 1)$ & ARC & yellow & TL95b-6677/10 \\
\hline Drought tolerant population two & DTP-2- $\mathrm{C}_{5}(\mathrm{~W} 2)$ & ARC & white & TL 95b-6677/11 \\
\hline Drought tolerant population two & DTP-2- $\mathrm{C}_{5}(\mathrm{Y} 2)$ & ARC & yellow & TL 95b-6677/12 \\
\hline
\end{tabular}

Agriculture Research Center (ARC). 
3-Leaf proline content; three fresh-leaf samples were taken for determining leaf proline content $(\mathrm{mg} / \mathrm{g})$ as physiological indicators of plant status under the implemented water stress treatments. Sampling time was at 65 days after planting representing flowering stages. Samples were collected between 11:00 am and 2:00 pm. Leaf disks were taken from two plants in each plot. The leaf disks were immersed immediately in the cooled proline extraction solution $(3 \%$ aqueous sulfosalicylic acid solution).

Samples were taken to cooled conditions and were kept in refrigerator until the extraction and determination of leaf proline content (Bates et al., 1973). Samples were measured by spectrophotometer and repeated twice.

\section{Statistical analysis}

Statistical analysis was performed according to steel and torrei (1982), by using ANOVA at SAS software (SAS. Software Rel 6.12, 1997). Water treatments and genotypes were treated as fixed effects, while replications as random effects. Treatments means were compared by $\mathrm{LSD}_{0.05}$ and calculated using SAS software. Test for homogeneity of error variances were carried out according to snedecor and chochran (1981). Heterogeneity differences were observed between the years error variances; therefore, theseparte analysis of variance for each year was done.

\section{RESULTS AND DISCUSSION}

The analysis of variance for grain yield, plant height and proline content in 2014 and 2016 seasons were recorded in Table (2). Highly significant variances were observed among water deficiency treatments for all the studied traits at the two tested seasons for all studied traits. The treatments by genotypes interaction were significant for the studied traits at the two seasons, except for grain yield at 2016 season (Table 2).

\section{Grain yield (ard/fad)}

Means of grain yield and the other studied traits of the evaluated 15 maize genotypes at three water stress treatment were presented in Table (3). the results showed that, water stress treatments affected on all studied traits, where, 10 days treatment gave the highest grain yield in 2014 and 2016 seasons (11.01 and $10.63 \mathrm{ard} / \mathrm{fad}$ ), respectively, 15 days treatments had significantly lower yield in 2014 season and insignificant difference in 2016 season (8.97 and 9.13 ard/fad),respectively. The 20 days water treatment had the least significant grain yield at both seasons (7.72 and $7.78 \mathrm{ard} / \mathrm{fad})$, respectively. Generally, the single crosses had more significant grain yield than the tested populations and lines, while the tested lines had the lowest significant grain yield (Table 4). Non-significant differences were observed among S.c. 10, 162 and Twc. 321 in 2014 season $(16.57,16.11$ and $15.88 \mathrm{ard} / \mathrm{fad}$ respectively), while at 2016 season Sc.10 had significantly more grain yield than the other crosses (16.82ard/fad). Also, Sc.162 and Twc.321had insignificant differences at 2016 season (15.18 and $14.55 \mathrm{ard} / \mathrm{fad})$, respectively, while Sc.129 was significantly lower yield (13.45ard/fad).

Table 2: Mean square of grain yield, plant height and proline content for 15 maize genotypes evaluated under stress water treatments in 2014 and 2016 seasons.

\begin{tabular}{lccccccc}
\hline \multirow{2}{*}{ S.O.v } & \multirow{2}{*}{ d.f } & \multicolumn{2}{c}{$\begin{array}{c}\text { Grain yield } \\
\text { (ard/fad) }\end{array}$} & \multicolumn{2}{c}{$\begin{array}{c}\text { Plant height } \\
(\mathbf{c m})\end{array}$} & \multicolumn{2}{c}{$\begin{array}{c}\text { Proline content } \\
(\mathbf{m g} / \mathbf{g})\end{array}$} \\
\cline { 2 - 7 } & & $\mathbf{2 0 1 4}$ & $\mathbf{2 0 1 6}$ & $\mathbf{2 0 1 4}$ & $\mathbf{2 0 1 6}$ & $\mathbf{2 0 1 4}$ & $\mathbf{2 0 1 6}$ \\
\hline Rep & 3 & 5.35 & 2.76 & 31.48 & 0.37 & 0.64 & 2.55 \\
\hline Trt & 2 & $165.57^{* *}$ & $121.44^{* *}$ & $12771.67^{* *}$ & $9196.25 * *$ & $7440.32^{* *}$ & $6929.30 * *$ \\
\hline Error a & 6 & 0.31 & 0.41 & 2.14 & 3.12 & 0.21 & 0.78 \\
\hline Gen & 14 & $200.28 * *$ & $180.82^{* *}$ & $16729.23^{* *}$ & $19367.21 * *$ & $525.38^{* *}$ & $531.02 * *$ \\
\hline Trt*gen & 24 & $2.33^{* *}$ & $0.99 \mathrm{~ns}$ & $226.43^{* *}$ & $631.67 * *$ & $69.10^{* *}$ & $67.87 * *$ \\
\hline Error & 126 & 0.84 & 0.98 & 5.39 & 7.95 & 0.75 & 2.14 \\
\hline$* * *$ significant and highly significant differences at 0.05 and 0.01 levels of probability & &
\end{tabular}

Table 3: Means of grain yield, plant height and proline content at three water stress during 2014 and 2016 seasons.

\begin{tabular}{lcccccc}
\hline & Character & \multicolumn{2}{c}{$\begin{array}{c}\text { Grain yield } \\
\text { (ard/fad) }\end{array}$} & \multicolumn{2}{c}{$\begin{array}{c}\text { Plant height } \\
(\mathbf{c m})\end{array}$} & \multicolumn{2}{c}{$\begin{array}{c}\text { Proline content } \\
\text { (mg/g) }\end{array}$} \\
\cline { 2 - 7 } treatment & $\mathbf{2 0 1 4}$ & $\mathbf{2 0 1 6}$ & $\mathbf{2 0 1 4}$ & $\mathbf{2 0 1 6}$ & $\mathbf{2 0 1 4}$ & $\mathbf{2 0 1 6}$ \\
\hline 10 days & $11.01 \mathrm{a}$ & $10.63 \mathrm{a}$ & $187.84 \mathrm{a}$ & $184.50 \mathrm{a}$ & $30.74 \mathrm{c}$ & $30.60 \mathrm{c}$ \\
\hline 15 days & $8.97 \mathrm{~b}$ & $9.13 \mathrm{a}$ & $172.50 \mathrm{~b}$ & $172.75 \mathrm{~b}$ & $41.63 \mathrm{~b}$ & $42.17 \mathrm{~b}$ \\
\hline 20 days & $7.72 \mathrm{~b}$ & $7.78 \mathrm{~b}$ & $158.67 \mathrm{c}$ & $159.75 \mathrm{c}$ & $53.01 \mathrm{a}$ & $52.07 \mathrm{a}$ \\
\hline $\mathrm{LSD}_{0.05}$ & 1.69 & 1.95 & 4.45 & 5.37 & 1.39 & 2.68 \\
\hline
\end{tabular}


As For, the populations, in significant differences were detected at 2014 season, while at 2016 season $\mathrm{W}_{2}$ and $\mathrm{Y}_{1}$ populations had significantly more grain yield (9.27 and $9.57 \mathrm{ard} / \mathrm{fad})$. Among tested lines, Sd.7 and Gz.653 lines had the highest significant grain yield at both seasons $(7.48,7.30$ and 6.84 , $7.41 \mathrm{ard} / \mathrm{fad}$, respectively), (Table 4). The interaction between water stress treatments and genotypes is shown in table $(5,6)$. Similar results were in agreement trend with those reported by Frederick et al. (1989)., Nigem (1998), Moursi (1997), Abd ElGawadet al. (1980), Moustafa and Seif El-Yazal (1980).

\section{Plant height $(\mathbf{c m})$}

Plant height was reduced when water stress treatments applied, where, 20 days treatment had the least plant heights at both seasons (158.67 and $159.75 \mathrm{~cm}$, respectively). The 15 days treatment had 172.50 and $172.75 \mathrm{~cm}$ of plant height at both seasons, respectively, (Table3). On the other hand, the tallest plants were observed for 10 days treatment (187.84 and $184.50 \mathrm{~cm}$, respectively). Sc.10 gave the tallest plants while Twc.321 had the lowest plant height than Sc.162 and Sc.129 in 2014 and 2016, (239.58 and $237.50 \mathrm{~cm}),(212.50$ and $213.75 \mathrm{~cm}),(221.25$ and $222.50 \mathrm{~cm}),(205.42$ and $204.17 \mathrm{~cm}$ ), respectively. For populations, $\mathrm{Y}_{2}$ had significant difference of plant height in 2014 season, while $\mathrm{Y}_{2}$ and $\mathrm{W}_{2}$ had significant difference in 2016 season (Table 4). For lines tested, Gz.653 and Sd.63 had significant difference in both seasons from the others, where Gz.653 line gave the highest values (182.50 and $183.75 \mathrm{~cm}$, respectively) but $\mathrm{Sd} .63$ line gave the lowest values (110.84 and $87.92 \mathrm{~cm}$, respectively). The interaction between water stress treatments and genotypes is shown in table $(5,6)$. Similar data was obtained by Porro and Cassel (1986), El-Ganayniet al. (2000) and Asch et al. (2001).

\section{Proline content $(\mathrm{mg} / \mathrm{g})$}

For proline content, the water stress treat-ments increased proline content in leaves, where the 20 days treatment had the highest values at both seasons (53.01 and $52.07 \mathrm{mg} / \mathrm{g}$ ). The 15 days treatment had 41.63 and $42.17 \mathrm{mg} / \mathrm{g}$ at both seasons, while, the least values of proline content were detected for 10 days water stress treatment (Table 3). Twc. 321 had the highest values among hybrids. On the other hand, Sc.10 gives the least values of proline content in leaves, $(50.45,51.03 \mathrm{mg} / \mathrm{g})$ and $(31.66,30.88 \mathrm{mg} / \mathrm{g})$ in 2014 and 2016 respectively. Population, $\mathrm{W}_{2}$ and $\mathrm{Y}_{2}$ had in significant differences in both seasons but showed highly values than the other popula-tions, where, population $\mathrm{W}_{1}$ gave the least val-ues in the two seasons, (Table 4). On the other hand Gm-2 line enjoyed the highest leaves proline content in both seasons (54.73and 53.81 $\mathrm{mg} / \mathrm{g}$, respectively), however Gz.639 line gave low values in the two seasons (32.35and $32.19 \mathrm{mg} / \mathrm{g}$, respectively). The interaction between water stress treatments and genotypes is shown in table $(5,6)$. Similar results were reported by Adel Soltani, et al (2013), Efeoĝlu,B. et al (2009), Mohsen Tarighaleslami, et al (2012), Lama, R. and Chakraborty, U. (2013), Heidari, Y. and Moaveni, P. (2009).

Table 4: Means of maize genotypes for grain yield, plant height and proline content at three water stress during 2014 and 2016 seasons.

\begin{tabular}{|c|c|c|c|c|c|c|}
\hline \multirow{3}{*}{ genotypes } & \multicolumn{2}{|c|}{$\begin{array}{c}\text { Grain yield } \\
\text { (ard/fad) }\end{array}$} & \multicolumn{2}{|c|}{$\begin{array}{c}\begin{array}{c}\text { Plant height } \\
(\mathrm{cm})\end{array} \\
\end{array}$} & \multicolumn{2}{|c|}{$\begin{array}{c}\text { Proline content } \\
(\mathrm{mg} / \mathrm{g})\end{array}$} \\
\hline & 2014 & 2016 & 2014 & 2016 & 2014 & 2016 \\
\hline & \multicolumn{6}{|c|}{ Lines } \\
\hline $\mathrm{Gm} 2$ & $5.37 \mathrm{e}$ & $5.41 \mathrm{f}$ & $124.58 \mathrm{~L}$ & $126.67 \mathrm{~J}$ & $54.73 a$ & $53.81 \mathrm{a}$ \\
\hline Sd 7 & $7.48 \mathrm{~d}$ & $7.30 \mathrm{e}$ & $144.58 \mathrm{~J}$ & $141.25 \mathrm{i}$ & $40.47 \mathrm{~g}$ & $40.12 \mathrm{e}$ \\
\hline Sd 63 & $6.42 \mathrm{~d}$ & $6.44 \mathrm{f}$ & $110.84 \mathrm{~m}$ & $87.92 \mathrm{k}$ & $44.42 \mathrm{~d}$ & $44.57 \mathrm{~d}$ \\
\hline Gz 612 & $5.57 \mathrm{e}$ & $5.76 \mathrm{f}$ & $168.34 \mathrm{~h}$ & $170.00 \mathrm{~g}$ & $37.91 \mathrm{~h}$ & $37.46 \mathrm{~g}$ \\
\hline $\mathrm{Gz} 628$ & $5.81 \mathrm{e}$ & $6.00 \mathrm{f}$ & $134.17 \mathrm{k}$ & $138.34 \mathrm{i}$ & $41.75 \mathrm{f}$ & $41.67 \mathrm{e}$ \\
\hline Gz 639 & $5.61 \mathrm{e}$ & $5.56 \mathrm{f}$ & $143.34 \mathrm{~J}$ & $141.67 \mathrm{i}$ & $32.35 \mathrm{i}$ & $32.19 \mathrm{~h}$ \\
\hline Gz 653 & $6.84 \mathrm{~d}$ & $7.41 \mathrm{e}$ & $182.50 \mathrm{f}$ & $183.75 \mathrm{f}$ & $46.87 \mathrm{c}$ & $47.44 \mathrm{c}$ \\
\hline \multicolumn{7}{|c|}{ Hybrid } \\
\hline Sc 10 & $16.57 \mathrm{a}$ & $16.82 \mathrm{a}$ & $239.58 \mathrm{a}$ & $237.50 \mathrm{a}$ & $31.66 \mathrm{i}$ & $30.88 \mathrm{~h}$ \\
\hline Sc 129 & $13.14 \mathrm{~b}$ & $13.45 \mathrm{c}$ & $205.42 d$ & $204.17 d$ & $43.32 \mathrm{e}$ & $43.17 \mathrm{~d}$ \\
\hline Sc 162 & $16.11 \mathrm{a}$ & $15.18 \mathrm{~b}$ & $221.25 b$ & $222.50 \mathrm{~b}$ & $40.47 \mathrm{~g}$ & $41.13 \mathrm{e}$ \\
\hline Twc 321 & $15.88 \mathrm{a}$ & $14.55 \mathrm{~b}$ & $212.50 \mathrm{c}$ & $213.75 \mathrm{c}$ & $50.45 \mathrm{~b}$ & $51.03 \mathrm{~b}$ \\
\hline \multicolumn{7}{|c|}{ Population } \\
\hline W 1 & $8.06 \mathrm{c}$ & $7.91 \mathrm{e}$ & $159.17 \mathrm{i}$ & $162.50 \mathrm{~h}$ & $31.87 \mathrm{i}$ & $32.09 \mathrm{~h}$ \\
\hline W 2 & $8.17 \mathrm{c}$ & $9.27 \mathrm{~d}$ & $186.25 \mathrm{e}$ & $189.17 \mathrm{e}$ & $45.53 \mathrm{~d}$ & $43.97 \mathrm{~d}$ \\
\hline Y 1 & $8.57 \mathrm{c}$ & $9.57 \mathrm{~d}$ & $173.34 \mathrm{~g}$ & $176.25 \mathrm{f}$ & $40.15 \mathrm{~g}$ & $39.52 \mathrm{f}$ \\
\hline Y 2 & $8.90 \mathrm{c}$ & $7.09 \mathrm{e}$ & $189.17 \mathrm{e}$ & $189.58 \mathrm{e}$ & $44.96 \mathrm{~d}$ & $45.17 \mathrm{~d}$ \\
\hline $\mathrm{LSD}_{0.05}$ & 1.27 & 1.37 & 3.22 & 3.90 & 1.20 & 2.03 \\
\hline
\end{tabular}


Table 5: Interaction between the water stress treatments and maize genotypes on grain yield,plant height and proline content during 2014seasons.

\begin{tabular}{|c|c|c|c|c|c|c|c|c|c|}
\hline \multirow{2}{*}{ genotypes } & \multicolumn{3}{|c|}{$\begin{array}{c}\text { Grain yield } \\
\text { (ard/fad) }\end{array}$} & \multicolumn{3}{|c|}{$\begin{array}{c}\text { Plant height } \\
(\mathrm{cm})\end{array}$} & \multicolumn{3}{|c|}{ Proline content $(\mathrm{mg} / \mathrm{g})$} \\
\hline & 10 & 15 & 20 & 10 & 15 & 20 & 10 & 15 & 20 \\
\hline $\mathrm{Gm} 2$ & 6.69 & 4.89 & 4.52 & 161.25 & 111.25 & 101.25 & 43.85 & 58.50 & 61.83 \\
\hline Sd 7 & 9.67 & 6.75 & 6.01 & 163.75 & 143.75 & 126.25 & 28.45 & 29.63 & 53.33 \\
\hline Sd 63 & 8.57 & 5.75 & 4.92 & 127.50 & 107.50 & 97.50 & 34.87 & 45.84 & 52.54 \\
\hline Gz 612 & 7.40 & 5.06 & 4.26 & 191.25 & 161.25 & 152.50 & 26.65 & 41.34 & 45.73 \\
\hline Gz 628 & 7.23 & 5.76 & 4.43 & 152.50 & 132.50 & 117.50 & 33.23 & 40.68 & 51.33 \\
\hline Gz 639 & 7.29 & 5.45 & 4.08 & 152.50 & 143.75 & 133.75 & 24.16 & 33.66 & 39.21 \\
\hline Gz 653 & 8.02 & 7.05 & 5.46 & 197.50 & 182.50 & 167.50 & 34.12 & 45.57 & 60.92 \\
\hline Sc 10 & 19.59 & 17.19 & 12.94 & 257.50 & 247.50 & 213.75 & 24.34 & 27.57 & 43.06 \\
\hline Sc 129 & 14.57 & 13.02 & 11.82 & 213.75 & 208.75 & 193.75 & 35.41 & 44.72 & 49.81 \\
\hline Sc 162 & 17.25 & 16.16 & 14.93 & 227.50 & 222.50 & 213.75 & 26.63 & 40.08 & 54.72 \\
\hline Twc 321 & 17.49 & 16.83 & 13.32 & 221.25 & 213.75 & 202.50 & 38.30 & 54.56 & 58.51 \\
\hline W 1 & 10.60 & 7.09 & 6.50 & 170.00 & 158.75 & 148.75 & 24.92 & 27.63 & 43.06 \\
\hline W 2 & 10.05 & 7.51 & 6.94 & 196.25 & 186.25 & 176.25 & 28.09 & 46.54 & 61.96 \\
\hline Y 1 & 10.68 & 7.84 & 7.18 & 182.50 & 173.75 & 163.75 & 26.93 & 33.71 & 59.81 \\
\hline Y 2 & 10.03 & 8.77 & 7.89 & 202.50 & 193.75 & 171.25 & 29.98 & 45.53 & 59.35 \\
\hline $\mathrm{LSD}_{0.05}(\mathrm{trt})$ & & 0.44 & & & 1.15 & & & 0.36 & \\
\hline $\mathrm{LSD}_{0.05}$ (gen) & & 0.73 & & & 1.86 & & & 0.69 & \\
\hline
\end{tabular}

Table 6: Interaction between the water stress treatments and maize genotypes on grain yield,plant height and proline content during 2016seasons.

\begin{tabular}{|c|c|c|c|c|c|c|c|c|c|}
\hline \multirow{2}{*}{ genotypes } & \multicolumn{3}{|c|}{$\begin{array}{c}\text { Grain yield } \\
\text { (ard/fad) }\end{array}$} & \multicolumn{3}{|c|}{$\begin{array}{l}\text { Plant height } \\
\text { (cm) }\end{array}$} & \multicolumn{3}{|c|}{$\begin{array}{l}\text { Proline content } \\
(\mathrm{mg} / \mathrm{g})\end{array}$} \\
\hline & 10 & 15 & 20 & 10 & 15 & 20 & 10 & 15 & 20 \\
\hline $\mathrm{Gm} 2$ & 6.57 & 5.04 & 4.60 & 162.50 & 113.75 & 103.75 & 43.09 & 58.67 & 59.67 \\
\hline Sd 7 & 9.05 & 7.07 & 5.78 & 158.75 & 141.25 & 123.75 & 29.58 & 41.83 & 48.96 \\
\hline Sd 63 & 8.64 & 5.58 & 5.09 & 85.75 & 106.25 & 98.75 & 33.61 & 46.61 & 53.50 \\
\hline Gz 612 & 7.52 & 5.40 & 4.36 & 192.50 & 163.75 & 153.75 & 26.91 & 39.68 & 45.79 \\
\hline $\mathrm{Gz} 628$ & 7.57 & 5.76 & 4.65 & 157.50 & 137.50 & 120.00 & 33.45 & 40.33 & 51.23 \\
\hline Gz 639 & 6.94 & 5.81 & 3.93 & 151.25 & 142.50 & 131.25 & 24.57 & 32.54 & 39.45 \\
\hline $\mathrm{Gz} 653$ & 8.22 & 7.37 & 6.62 & 201.25 & 183.75 & 166.25 & 37.01 & 45.24 & 60.06 \\
\hline Sc 10 & 18.71 & 17.47 & 14.28 & 258.75 & 245.00 & 208.75 & 23.75 & 27.45 & 41.45 \\
\hline Sc 129 & 14.83 & 13.68 & 11.84 & 212.50 & 207.50 & 192.50 & 33.85 & 46.12 & 49.54 \\
\hline Sc 162 & 16.90 & 15.02 & 13.62 & 232.50 & 222.50 & 212.50 & 26.03 & 41.72 & 55.63 \\
\hline Twc 321 & 16.30 & 14.54 & 13.06 & 222.50 & 211.25 & 207.50 & 38.82 & 55.11 & 59.15 \\
\hline W 1 & 9.39 & 7.49 & 6.83 & 171.25 & 160.00 & 156.25 & 25.35 & 28.76 & 42.16 \\
\hline W 2 & 10.33 & 9.84 & 7.63 & 201.25 & 188.75 & 177.50 & 26.60 & 48.23 & 57.10 \\
\hline Y 1 & 10.41 & 9.61 & 8.70 & 183.75 & 175.00 & 170.00 & 26.50 & 32.86 & 59.20 \\
\hline Y 2 & 8.27 & 7.26 & 5.75 & 202.50 & 192.50 & 173.75 & 29.89 & 47.44 & 58.17 \\
\hline $\mathrm{LSD}_{0.05}$ (trt) & & 0.51 & & & 1.39 & & & 0.69 & \\
\hline $\operatorname{LSD}_{0.05}$ (gen) & & 0.79 & & & 2.26 & & & 1.17 & \\
\hline
\end{tabular}

\section{REFERENCES}

Abd El-Gawad, A.A., El-Tabbakh, A. and Mahgoub, G.A. (1980). Yield and sink capacity response of maize plants to drought and nitrogen fertilization. Egypt. J. agron. 5: 1-13.

Asch, F., Andersen, M.N., Jensen, C.R. and Mogensen, V.O. (2001). Ovary abscisic acid concentration does not induce kernel abortion in field-grown maize subjected to drought. Europ.J. of Agron. 15: 119-129.
Bates, L.S. (1973). Rapid determination of free proline for water-stress studies. Plant and soil, 39: 205-207.

Efeoĝlu ${ }^{\mathrm{a}}$ B., Ekmekçi ${ }^{\mathrm{b},{ }^{*}}$ Y., Çiçek ${ }^{\mathrm{b}} \mathrm{N}$. (2009). Physiological responses of three maize cultivars to drought stress and recovery. South African Journal of Bo-tany 75 (2009) 34-42. 
El-Ganayni, A.A., A.M. Al-Naggar, H.Y. ElSherbieny, and M.Y. El-Sayed (2000). Genotypic differences among 18 maize populations in drought tolerance at differ-ent growth stages. J.Agric. Sci. Mansoura Univ. 25: 713-727.

Frederick,J.R.;J.D.Hesketh,;D.B.Peters and F.E. Below, (1989).Yield and rep-roductive trait responses of maize hybrids to drought stress. Maydica, 34(4): 319 - 328.

Heidari,Y. and Moaveni,P. (2009). Study of drought stress on aba accumulation and proline among in different genotype for-age corn. Research journal of biological sciences 4 (10): 11211124, 2009.

Howell, T. A., A. Yazar, A. D. Schneider, D. A. Dusek, and K. S. Copeland (1995). Yield and water use efficiency of maize in resp-onse to LEPA irrigation. Trans. ASAE 38, 1737-1747.

Huang.J,Hirji.R,Adam.L,Rozwadowski.KL,Hamme rlindl.JK,Keller.WAandSelvara.jG(2000).

Genetic engineering of glycinebet-aine production toward enhancing stress tolerance in plants: metabolic limitations, Plant Physiol. 122:747-756.

Kavikishore PB, Sangam S, Amrutha RN, Srilaxm P, Naidu KR, Rao KRSS, RaoS,ReddyKJ,TheriappanP,Sreenivasulu N (2005). Regulation of proline biosynthe-sis, degradation, uptake and transport in higher plants: its implications in plant growth and abiotic stress tolerance, Curr. Sci. 88: 424438.

Lama, R. and U.Chakraborty, (2013). Water Deicit Stress Tolerance Traits in Maize (Zea mays L.) and Ideniicaion of Tolerant Varieies. International Jou-rnal of Bio-resource and Stress Mana-gement 2013, 4(2) special: 328333.

Moursi, A. M. (1997).Studies on drought tolera-nce in maize. M. Sc. Thesis, Fac. Agric., Zagazig Univ., Egypt.
Moustafa, A.T.A. and Seif-El-Yazal, M.N. (1980). Effect of irrigation frequency at different stages of growth on corn grain yield and its content of N, P, and K. Agric. Res. Rev. 58: 179-185.

NeSmith, D. S., and J. T. Ritchie (1992). Short- and long-term responses of mai-ze to a pre-anthises soil water stress. Agron. J. 84, 107-113.

Nigem, S. A. (1989). Varietal response to nitrogen fertilization in maize.J.Appl. Sci., 4: 127 - 139

Porro, I. and Cassel, D.K. (1986).Response of corn to tillage and delayed irriga-tion. Agron. J. 78: 688-693.

Rhoads, F. M., and J. M. Bennett (1990). Maize. In: B. A. Stew- art, and D. R. Nielsen, eds. Irrigation of Agric-ultural Crops, pp. 569-596. American Society of Agronomy, Madison, WI.

SAS 1997. SAS software Rel. 6.12, SAS Inst.,Cary, NC. USA.

Snedecor,G.W. and W.G.Chochran, (1981) .Statistical Method, $7^{\text {th }}$ Ed., lowa State Univ. Press, Ames.lowa, USA.

Soltani, A. A. Waismoradi, M. Heidari and H. Rahmati ${ }^{1}$.(2013). Effect of Water Stress and Nitrogen on Yield and Compatibility Metabolites on Two Medium Maturity Corn Cultivars. Interna-tional Journal of Agriculture and Crop Sciences. IJACS/ 2013/5-7/737-740.

Steel ,R.G.D. and T.H.Torrie (1982). Prin-ciples and procedures of statistics, Mc-Graw hill. International book company, london, 633.

Stone, P. J., D. R. Wilson, J. B. Reid, and G. N. Gillespie (2001). Water de?cit effects on sweet maize: I. Water use, radiation use efficiency, growth, and yield. Aust. J. Agric. Res. 52, 103113.

Tarighaleslami, M.R. Zarghami, M. Mashhadi, A. Boojar and M. Oveysi.(2012). Effects of Drought Str-ess and Different Nitrogen Levels on Morphological Traits of Proline in Le-af and Protein of Corn Seed (Zea mays L.). AmericanEurasian J. Agric. \& Environ. Sci., 12 (1): 4956, 2012 . 


\section{المالهص المري}

العلاقة بين الإجهاد المائى ومحصول الحبوب، إرتفاع النبات، كمية البرولين فى الذرة الثامية

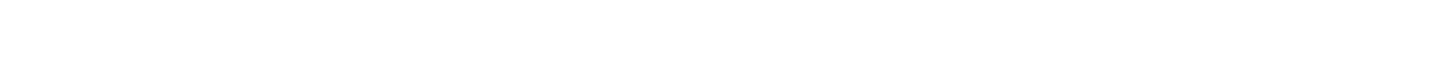

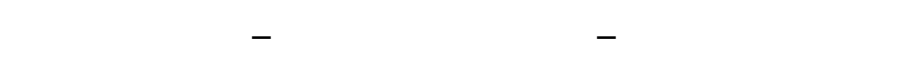

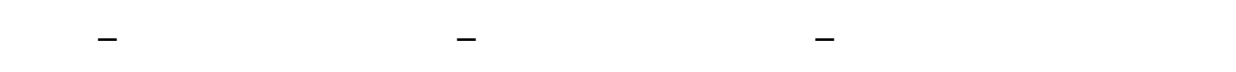

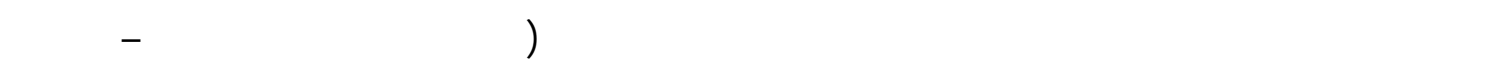

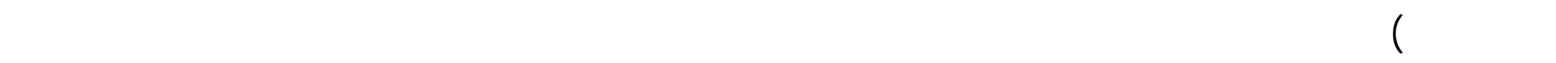

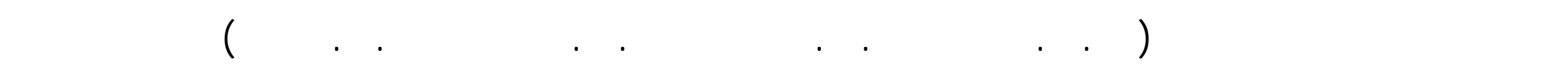

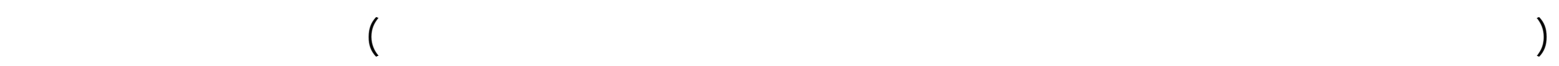

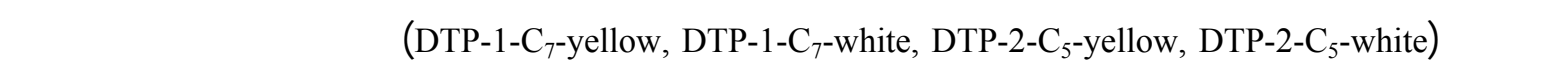
عله محصول الحبوب ولططول النباتلت وكذك قيلس محتوى الأوراق من البرولين.

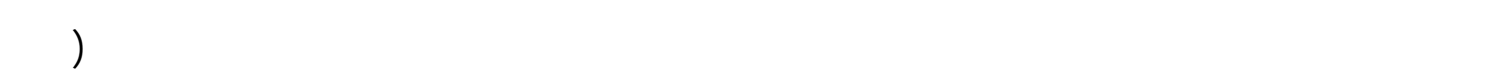

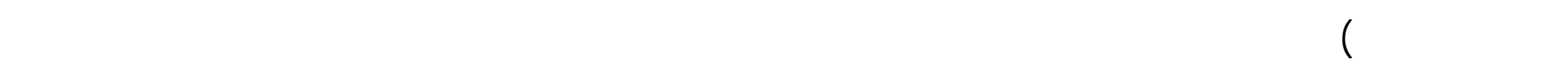

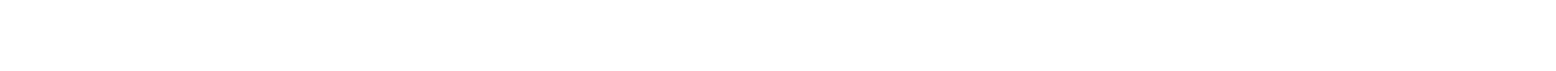
الحلمض الأميف البرولين خلال موسمى الدرلمادة.

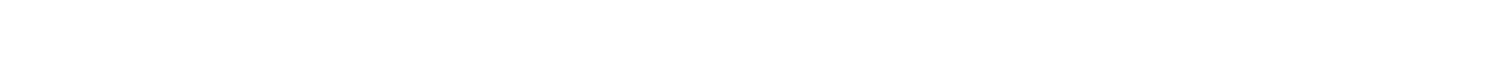

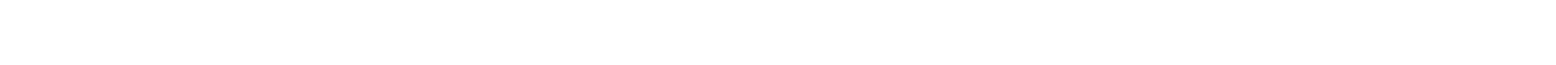

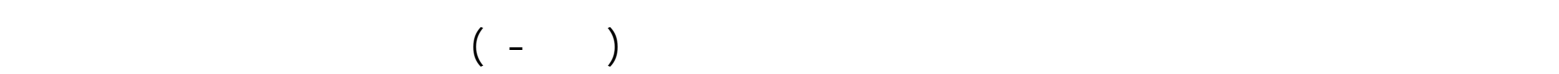
محصول خلل الموسمين اليضاً.

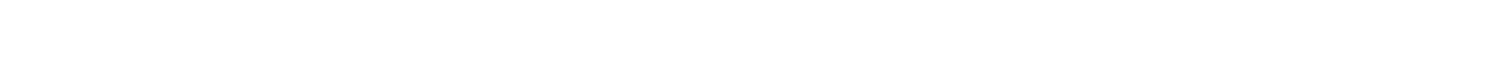

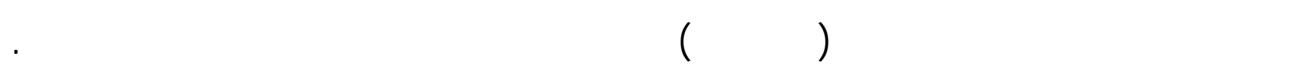

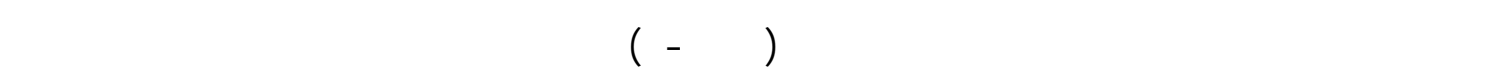

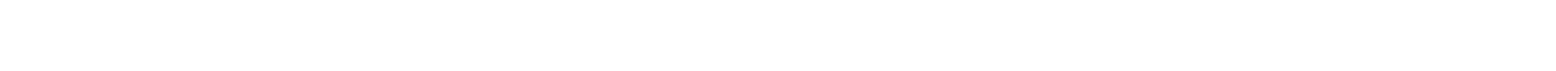

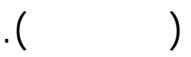

